\title{
CHIP-SCALE ATOMIC DEVICES
}

\author{
John Kitching, Svenja Knappe, Peter D. D. Schwindt, Ying-Ju Wang, Hugh Robinson and Leo Hollberg \\ Time and Frequency Division, National Institute of Standards and Technology \\ Boulder, CO USA
}

\author{
Li-Anne Liew, John Moreland \\ Electromagnetics Division, \\ National Institute of Standards and Technology \\ Boulder, CO USA
}

\author{
Alan Brannon, Jason Breitbarth, Brad \\ Lindseth and Zoya Popovic \\ Department of Electrical and Computer \\ Engineering, The University of Colorado \\ Boulder, CO USA
}

\begin{abstract}
We present an overview of recent work to develop chipscale atomic devices such as frequency references and magnetometers. These devices take advantage of advances over the last ten years in the fields of micro electro mechanical systems (MEMS), precision atomic spectroscopy and semiconductor lasers. The convergence of processes and techniques from these three disparate areas allows for highly compact, low-power sensors with exceptional sensitivity and stability. We will discuss in detail several important ongoing activities in our laboratory including recent advances in alkali cell fabrication and new physics package designs for chip-scale atomic clocks and magnetometers. Finally, we discuss opportunities related to the coupling of resonant, magnetic microstructures to atoms for highly compact, low power sensor applications.
\end{abstract}

\section{INTRODUCTION}

Instruments based on precisely measured transitions in atoms include clocks [1], magnetometers [2, 3], gyroscopes [4, 5], accelerometers [6] and RF power meters [7]. Many of these instruments provide, or have the potential to provide, the most sensitive measurements of basic quantities (time, magnetic field, angular rotation rate, linear acceleration) available. However, in their most precise form, they are typically large and complex, and require several person-years of effort to construct. A key challenge in moving these instruments into widespread use in applications is miniaturization, and the associated gains in power consumption and cost. MEMS micromachining appears to provide an excellent avenue for achieving this miniaturization and enabling precision instrumentation in the increasing spectrum of portable, batteryoperated systems.

The development of chip-scale atomic clocks, for example, has progressed rapidly from concept [8] to component-level demonstration [9] to subsystem demonstration [10, 11] to prototype [12]. Physics packages for chip-scale atomic magnetometers have also been demonstrated [13] and it is anticipated that even more atom-based sensors and instruments could be miniaturized using MEMS technology. We describe here one possible approach that may find general use in achieving these goals.

\author{
Vishal Shah \\ Department of Physics, The University of Colorado \\ Boulder, CO USA \\ Vladislav Gerginov \\ Department of Physics, University of Notre Dame \\ Notre Dame, IN USA \\ Matthew Eardley \\ Department of Physics, The State University of New York, \\ Stony Brook, NY USA
}

\begin{abstract}
Atoms in the gas phase hold a somewhat unique position in the world of precision measurement because of several important properties. First they are fairly simple quantum mechanical systems, with energy spectra composed of a few well-separated states rather than a continuum found in many solid-state systems. Particularly important for spectroscopic purposes are alkali atoms, which have a single valence electron. Because high interaction energies are required to perturb a closed electron shell, almost all properties of the atoms are determined by the position and orientation of the valence electron with respect to the nucleus.
\end{abstract}

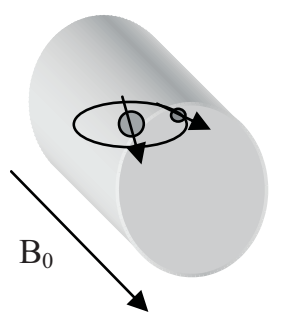

(a)

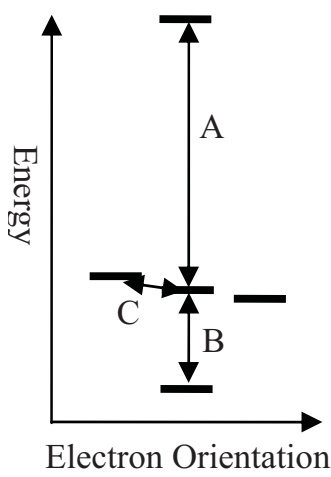

(b)
Figure 1. (a) The internal state of the atom and orientation in an external magnetic field $\left(B_{0}\right)$ determine its energy. (b) Transitions between energy levels (not to scale in the figure) may be induced by optical (A), microwave $(B)$ and $R F(C)$ electromagnetic fields.

We may consider a somewhat generic situation in which atoms at room temperature are confined in an otherwise evacuated volume and subjected to an external magnetic field (see Figure 1). In this case, four properties of the valence electron in the atom almost completely determine the energy spectrum: the mean distance of the electron from the nucleus, its orbital angular momentum about the nucleus, the orientation of the electron spin with respect to the nuclear spin and the orientation of the electron spin with respect to the external magnetic field. Transitions between these electron states can be excited by electromagnetic 
fields with a frequency equal to the energy difference between the states divided by Planck's constant, $h$. Typically, transitions between states involving the electron-nucleus distance and orbital angular momentum are excited by optical fields with frequencies above $100 \mathrm{THz}$. Transitions between orientations of the electron spin with respect to the nuclear spin are excited with microwave fields in the 1-10 GHz range. And transitions between orientations of the electron spin with the external magnetic field are induced with RF fields below $1 \mathrm{MHz}$, for magnetic fields less than $100 \mu \mathrm{T}$.

Alkali atoms in the gas phase can be made to interact only weakly with their surroundings; the random perturbations of the atomic energy levels in this case are rather small. As a result, the level energies are very well defined, as are the frequencies of the electromagnetic fields that connect any two levels. It is therefore possible to obtain detailed information about the internal state of the atom by measuring the frequency of the transition precisely. Alternatively, the universal and long-lived nature of the internal states can be used to stabilize the frequency of an external oscillator and make a precise and/or accurate frequency standard.

\section{MEMS ALKALI CELL FABRICATION}

Perhaps the most significant technological advances so far in enabling MEMS-based atomic devices are methods for fabricating highly miniaturized evacuated cells containing vapors of alkali atoms. The difficulty here is in maintaining a very pure environment inside the cell. This is required to prevent contaminants from reacting with the alkali atoms and from perturbing their energy levels. Traditionally, vapor cells for almost all atom-based instruments have been fabricated either with ultrahigh vacuum technology or by using glass-blowing techniques, in which discrete glass elements are fused and shaped by heating the material to near its softening point. The difficulties with this process are that the resulting cells tend to be fairly large, difficult to integrate with other components, and are usually made one-byone resulting in high cost.

MEMS-based assembly processes promise to revolutionize the way in which alkali atom cells are fabricated and used. Perhaps the simplest design [9], is shown in Figure 2. In this design, a hole is etched in a Silicon wafer and glass wafers are bonded to the top and bottom surfaces, forming an empty cavity. Alkali atoms are then confined in this sealed volume, along with a controlled environment such as a pure buffer gas, which lengthens the coherence time of the alkali oscillating moment by preventing frequency collisions with the cell walls.

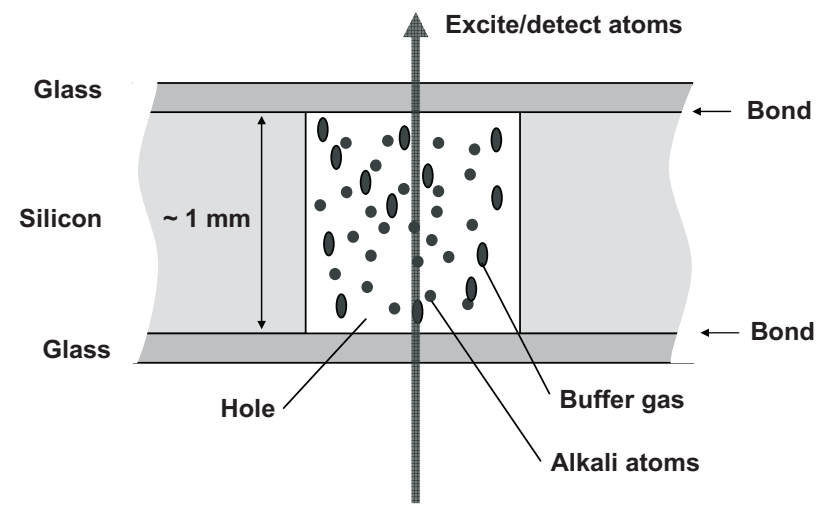

Figure 2. Design of a MEMS-based alkali atom vapor cell for chip-scale atomic devices.

The processes by which alkali atoms are introduced into the cell vary substantially. Pure alkali elements are metallic substances for which a vapor phase coexists with either a solid or liquid phase at room temperature. The atoms in the vapor phase are those that have the properties necessary for precision spectroscopy. But since the vapor phase is present whenever the solid or liquid exists, Alkali material can be directly deposited into cell as a solid or liquid under a controlled environment such as that produced inside a glove box or anaerobic chamber $[9,11,14]$.

It is also possible to fill the cells in a manner similar to conventional glass-blowing, in which a filling tube is connected to the cell interior through a hole in one of the glass surfaces $[15,16]$. The cell can then be evacuated with a vacuum pump and alkali atoms distilled into the cell with thermal gradients. A third method involves a chemical reaction to produce the pure alkali metal. This reaction can be carried out either inside the cell before it is bonded [9] or in an external ampoule with an opening placed near the cell opening [17]. Waxes have also been used to seal cells closed once the alkali atoms have been distilled into the interior volume [18]. Compact systems for confining laser cooled atoms at microKelvin temperatures are also under development [19].

A new technique being developed at NIST that shows considerable promise for wafer-level batch fabrication is the evaporative deposition of $\mathrm{CsN}_{3}$ into the cell preform, followed by ultra-violet dissociation of the azide material into Cs and $\mathrm{N}_{2}$ after sealing [20]. This technique allows a careful control of the final buffer gas $\left(\mathrm{N}_{2}\right.$, in this case) pressure in the cell through the adjustment of the time the cell is subject to the UV light. This could be used to advantage in fabricating temperaturecompensated cells with two or more buffer gases. Finally, a technique based on diffusion of alkali atoms through the glass windows at high temperature has is also under development [21], which may enable very low-cost cell production.

A common advantage of all of these schemes, in addition to the capability for small sizes, is the possibility of fabricating large numbers of vapor cells with the same process sequence on a single wafer. A conceptual view of how a final wafer of cells might look in shown in Figure 3.

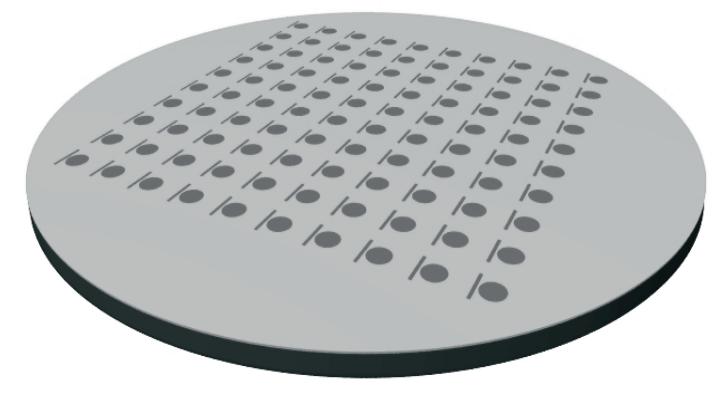

Figure 3. Conceptual implementation of wafer-level cell fabrication.

\section{PHYSICS PACKAGE DESIGNS}

The integration of the MEMS vapor cells with miniature optical components provides an interface that connects the atomic states and coherent oscillation with the outside world. Transitions in the atoms are excited with a modulated laser field [22], which can be decomposed into components of an optical spectrum that differ in frequency by the modulation frequency. This combination of spectral components allows a wide range of atomic transitions to be excited. A single spectral component, tuned into resonance with an optical transition in the atoms, will excite transitions between atomic energy levels with different electron-nucleus 


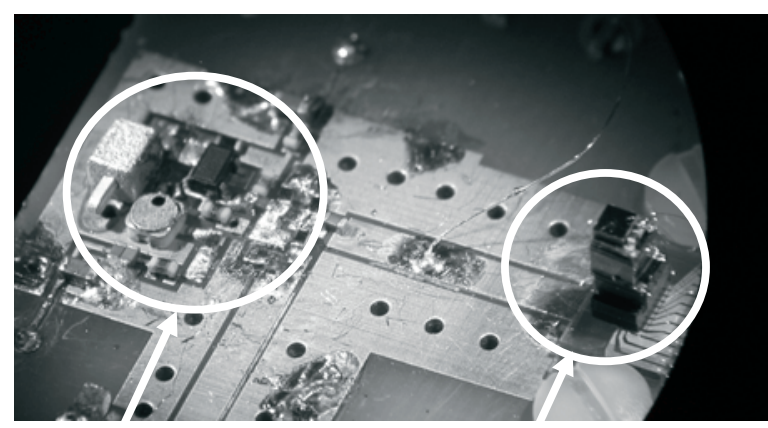

Local Oscillator

Physics Package

(a)

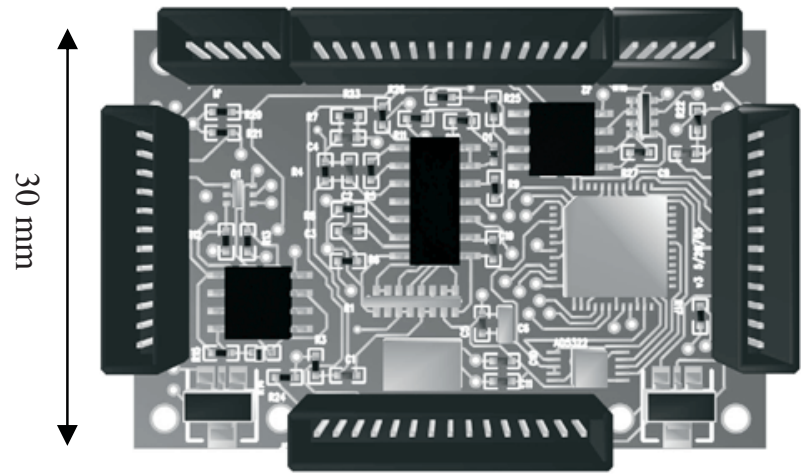

(b)

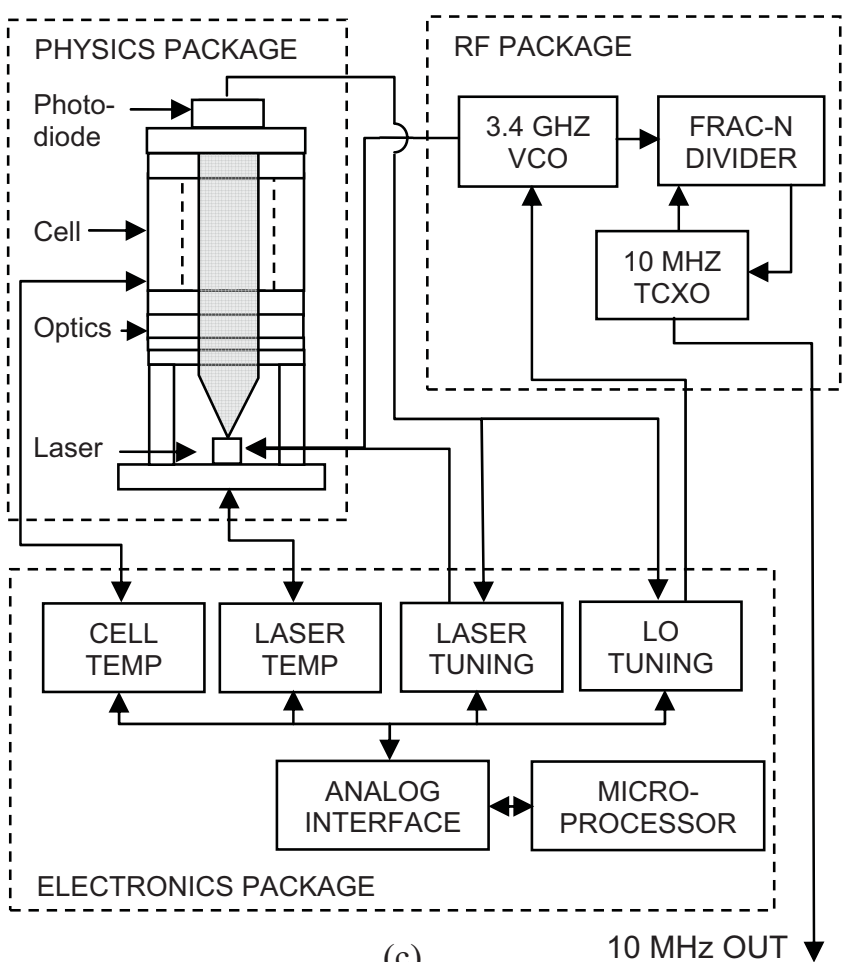

(c)

Figure 4. (a) Chip-scale atomic clock physics package and local oscillator integrated on a single substrate. (b) Control electronics design. (c) Schematic indicating major CSAC subsystems and interconnections. separation and/or orbital angular momentum. A combination of two spectral components can excite microwave and RF transitions in atoms through the fundamental nonlinearity of atomic systems [23]. A nonlinear resonator, driven by two fields with differing frequencies, can be made to oscillate at the sum or difference of those frequencies. Parametric amplification is one example of this process. When atoms are illuminated by two optical fields therefore, a coherent oscillation can be excited at the difference between the frequencies of those fields; the amplitude of this oscillation is particularly large if the difference frequency corresponds to a natural resonance in the atoms.

A typical physics package, therefore, consists of a modulated diode laser, some optics to adjust the light intensity, polarization and divergence to suitable values, a vapor cell, and a photodetector, that detects the transmitted optical power [10, 13]. A geometry typical of most of NIST's physics packages is shown in Figure 4(c). The physics package in this case is a passive device: it takes as its input a signal from an external oscillator and generates an output that depends on the difference between the frequency of the input signal and that of the atomic resonance.

NIST has recently demonstrated a chip-scale atomic clock physics package integrated with a compact, low-power oscillator based on a micro-coaxial resonator. Both the physics package [10] and local oscillator [24] are similar in structure to ones described previously. The integrated subsystems are shown schematically in Figure 4(a). The atomic resonance, as measured by the modulated diode laser, is shown in Figure 5(a) and the stability of the compact local oscillator, both when it is free running and when it is locked to the physics package, is shown in Figure 5(b). A compact control system has also been designed; the layout is shown in Figure 4(b). It is based on a microprocessor, which implements the four main servo systems required to run the clock. These servo systems actively stabilize the laser temperature, the cell temperature, the laser frequency (optical tuning) and the LO frequency. The microprocessor is interfaced with the physics package and local oscillator through an analog interface circuit. The main electrical connections between the different subsystems are shown in Figure 4(c).

Complete chip-scale atomic clock prototypes, which include not only the physics package and local oscillator but also compact implementations of the control system needed to run them have been demonstrated recently [12]. These devices have a volume near $10 \mathrm{~cm}^{3}$, require approximately $200 \mathrm{~mW}$ of electrical power to operate and achieve a short-term fractional frequency instability of a few parts in $10^{10}$ at one second of integration. While the longterm frequency instability has not been well characterized in these systems, a recent experiment has demonstrated that MEMS-based alkali vapor cells are capable of supporting an instability below $10^{-11}$ at one hour of integration and $10^{-10}$ at one day [17]. It appears likely that commercial systems with the specifications above and with steerable $10 \mathrm{MHz}$ output will be available in the 1-2 year timeframe. This will represent an improvement by an order of magnitude in size and by over an order of magnitude in power dissipation from the current state of the art in compact atomic clocks.

Further miniaturization to $1 \mathrm{~cm}^{3}$ and power reduction to near $30 \mathrm{~mW}$ also seems feasible. Physics packages dissipating less than $10 \mathrm{~mW}$ of power [11] and local oscillators dissipating below 5 $\mathrm{mW}$ have already been demonstrated [24]. It remains to develop a suitable compact, low-power control system and integrate all subsystems together.

Chip-scale atomic magnetometers have recently emerged as another type of sensor enabled by MEMS-based alkali cell fabrication. While atomic clocks are based on atomic transitions 
that are first-order insensitive to magnetic fields, chip-scale magnetometers use transitions whose frequencies depend on magnetic field. The first chip-scale magnetometer, demonstrated at

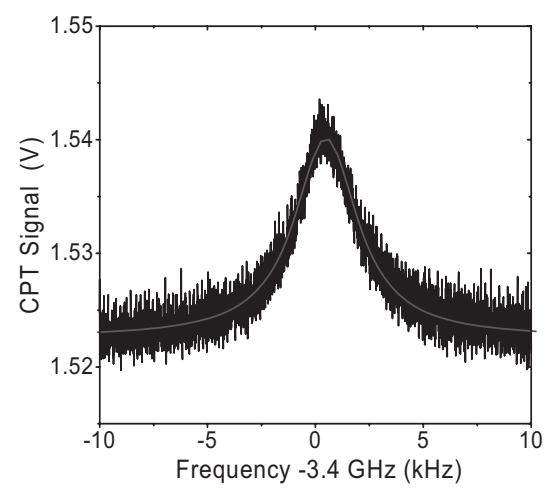

(a)

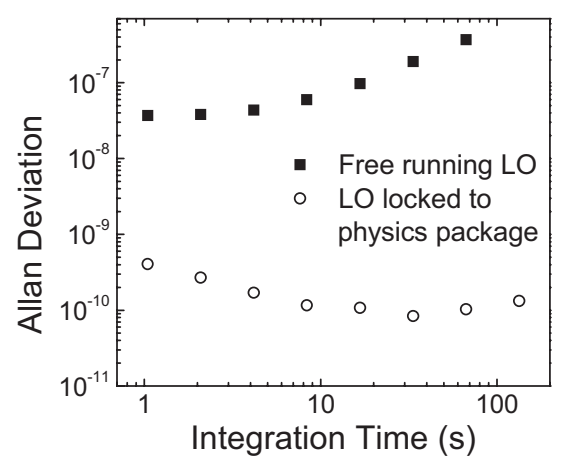

(b)

Figure 5. (a) Atomic resonance excited with a modulated diode laser. The resonance width is $3 \mathrm{kHz} F W H M$ based on the modulation frequency of $3.4 \mathrm{GHz}$ (b) The fractional frequency instability, as measured by the Alan deviation, as a function of integration time for the free-running local oscillator and one locked to the chip-scale physics package.

NIST in 2004 [13], achieved a sensitivity of $40 \mathrm{pT} / \sqrt{\mathrm{Hz}}$ at a frequency of $10 \mathrm{~Hz}$. Subsequent table-top experiments have shown that magnetic sensors incorporating MEMS-based vapor cells can achieve a sensitivity near $1 \mathrm{pT} / \sqrt{\mathrm{Hz}}$ at $10 \mathrm{~Hz}$. This sensitivity is comparable to that achieved by fluxgate sensors but the atombased device offers the advantages of being non-magnetic and of measuring the magnitude of the field, rather than the component along a single axis. The scalar nature of atomic magnetometers makes them largely insensitive to rotation and therefore suitable for use on moving or vibrating platforms.

The magnetometer described in Ref. [13] had a bandwidth of approximately $20 \mathrm{~Hz}$ that was limited by magnetic fields produced by the thin-film resistive heaters used to heat the cell to its operating temperature. The current running through the heaters was modulated at $40 \mathrm{~Hz}$ to move the effects of the associated magnetic field out of the low-frequency band where the sensor operated. However, considerable improvement in the bandwidth could be obtained with a MEMS-based cell heater that generated a magnetic field below $1 \mathrm{pT}$ while producing $50 \mathrm{~mW}$ of heat from a $3.3 \mathrm{~V}$ supply.

\section{DIRECTLY COUPLED ATOM-MEMS SYSTEMS}

In the work described above, MEMS techniques are used primarily for confining the atoms to a small volume. However, it is also conceivable that the quantum states of atoms in a vapor could be altered or probed with a MEMS device directly. Consider, for example, the experimental arrangement shown in Figure 6. Here a mechanically resonant microcantilever with a magnetic tip is coupled to an ensemble of atomic spins in an external magnetic field through their collective magnetic moment. Both the cantilever and the atoms have independent resonant frequencies: the cantilever's is determined by its geometry and material and the atoms' is determined by their magnetic moment and the applied DC magnetic field. If the cantilever is caused to oscillate at its resonant frequency, the motion of the cantilever tip causes an oscillating magnetic field at the location of the atoms. If the DC magnetic field is such that the atomic precession frequency (typically $\sim 10 \mathrm{GHz} / \mathrm{T}$ for alkali atoms) is equal to the frequency of the cantilever motion, this oscillating magnetic field can excite a coherent spin precession in the atoms. Alternatively, if the atoms are caused to precess by some other means, the cantilever can act as a very sensitive detector for the atomic motion. It may even be possible for the coupled resonator/atom system to oscillate on its own if the coupling could be made strong enough. Such a possibility was investigated theoretically Bargatin and Roukes in Ref. [25].

In some ways this idea is similar to recent work on the detection of single atomic spins embedded in solids using magnetic resonance force microscopy [26]. While the spatial resolution of this type of detection is superior in solid-state systems due to the highly localized nature of the spin, it may be that longer coherence times can be attained in atomic vapors due to the very weak interaction of atoms in the vapor phase with their surroundings. We anticipate that a device such as that shown in Figure 6 could be used as a very compact, sensitive magnetometer by comparing the atomic precession frequency, which is proportional to the magnetic field, to the cantilever resonant frequency.

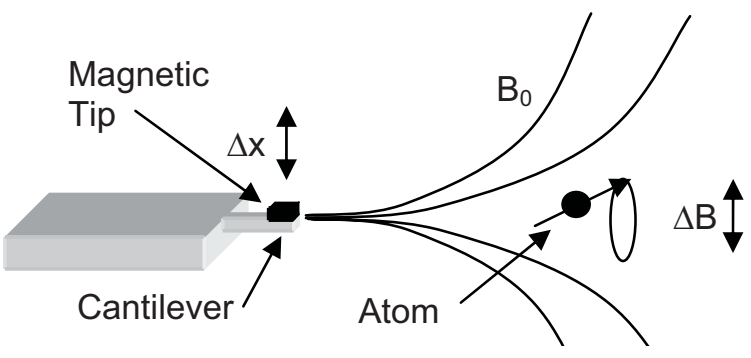

Figure 6. Atomic spins coupled directly to the motion of a micro-cantilever with a magnetic tip through their magnetic moment.

\section{CONCLUSIONS}

We have described in this paper an overview of some recent efforts to develop MEMS-based devices based on precise atomic transitions. The use of MEMS fabrication techniques offers the potential for small size, low power dissipation and parallel processing of many units for low cost production. Chip-scale atomic clocks are well under development. Most of the underlying physics and fabrication issues have been addressed and commercial devices are expected to appear soon. Physics packages for chip-scale atomic magnetometers, utilizing the same cell fabrication technology and with sensitivities in the range of $1 \mathrm{pT} / \sqrt{ } \mathrm{Hz}$ also seem feasible. Initial demonstration devices have yielded promising results. Finally, a scheme for the direct excitation and/or detection of atomic spins with a magnetic, mechanically resonant microstructure is proposed. 


\section{ACKNOWLEDGEMENTS}

This work is a contribution of NIST, an agency of the US Government, and is not subject to copyright. This work was supported by NIST and the Microsystems Technology Office of the Defense Advanced Research Projects Agency (DARPA).

\section{REFERENCES}

[1] J. Vanier and C. Audoin, The Quantum Physics of Atomic Frequency Standards. Bristol: Adam Hilger, 1992, and references therein.

[2] A. Bloom, "Principals of Operation of the Rubidium Vapor Magnetometer", Applied Optics, 1, 61 (1962).

[3] J. C. Allred, R. N. Lyman, T. W. Kornack, and M. V. Romalis, "High-Sensitivity Atomic Magnetometer Unaffected by Spin-Exchange Relaxation", Physical Review Letters, 89 (2002).

[4] J. T. Fraser, "Optically Pumped Magnetic Resonance Gyroscope and Direction Sensor," US Patent \#3,103,621, 1963.

[5] T. L. Gustavson, P. Bouyer, and M. A. Kasevich, "Precision Rotation Measurements with an Atom Interferometer Gyroscope", Physical Review Letters, 78, 2046 (1997).

[6] M. J. Snadden, J. M. McGuirk, P. Bouyer, K. G. Haritos, and M. A. Kasevich, "Measurement of the Earth's Gravity Gradient with an Atom Interferometer-Based Gravity Gradiometer", Physical Review Letters, 81, 971 (1998).

[7] J. C. Camparo, "Atomic Stabilization of Electromagnetic Field Strength Using Rabi Resonances", Physical Review Letters, 80, 222 (1998).

[8] J. Kitching, S. Knappe, and L. Hollberg, "Miniature Vapor-Cell Atomic-Frequency References", Applied Physics Letters, 81, 553 (2002).

[9] L. A. Liew, S. Knappe, J. Moreland, H. Robinson, L. Hollberg, and J. Kitching, "Microfabricated Alkali Atom Vapor Cells", Applied Physics Letters, 84, 2694 (2004).

[10] S. Knappe, V. Shah, P. D. D. Schwindt, L. Hollberg, J. Kitching, L. A. Liew, and J. Moreland, "A Microfabricated Atomic Clock", Applied Physics Letters, 85, 1460 (2004).

[11]R. Lutwak, J. Deng, W. Riley, M. Varghese, J. Leblanc, G. Tepolt, M.Mescher, D. K. Serkland, K. M. Geib, and G. M. Peake, "The Chip-Scale Atomic Clock - Low-Power Physics Package," Technical Digest of 36th Annual Precise
Time and Time Interval (PTTI) Meeting, Washington, DC, December 7-9 (2004), pp. 339.

[12] R. Lutwak, P. Vlitas, M. Varghese, M. Mescher, D. K. Serkland, and G. M. Peake, "The Mac - a Miniature Atomic Clock," Technical Digest of Joint IEEE International Frequency Control Symposium and Precise Time and Time Interval (PTTI) Systems and Applications Meeting Vancouver, Canada, August 29-31 (2005), pp. 752.

[13]P. D. D. Schwindt, S. Knappe, V. Shah, L. Hollberg, J. Kitching, L. A. Liew, and J. Moreland, "Chip-Scale Atomic Magnetometer", Applied Physics Letters, 85, 6409 (2004).

[14] M. H. Kwakernaak, S. Lipp, S. McBride, P. Zanzucchi, W. K. Chan, V. B. Khalfin, H. An, J. R. D. Whaley, B. I. Willner, A. Ulmer, J. Z. Li, T. Davis, A. M. Braun, J. H. Abeles, A. Post, Y.-Y. Jau, N. N. Kuzma, and W. Happer, "Components for Batch-Fabricated Chip-Scale Atomic Clocks," Technical Digest of 36th Annual Precise Time and Time Interval (PTTI) Meeting, Washington, DC, December 7-9 (2004), pp. 355.

[15] R. Lutwak, D. Emmons, T. English, W. Riley, A. Duwel, M. Varghese, D. K. Serkland, and G. M. Peake, "The Chip-Scale Atomic Clock - Recent Development Progress," Technical Digest of 35th Annual Precise Time and Time Interval (PTTI) Meeting, San Diego, CA, December 2-4 (2003), pp. 467.

[16] M. Zhu, L. S. Cutler, J. E. Berberian, J. F. DeNatale, P. A. Stupar, and C. Tsai, "Narrow-Linewidth Cpt Signal in Small Vapor Cells for Chip Scale Atomic Clocks," Technical Digest of IEEE International Frequency Control Symposium, Montreal, PQ, August 23-27 (2004), pp. 100.

[17] S. Knappe, V. Gerginov, P. D. D. Schwindt, V. Shah, H. G. Robinson, L. Hollberg, and J. Kitching, "Atomic Vapor Cells for Chip-Scale Atomic Clocks with Improved Long-Term Frequency Stability", Optics Letters, 30, 2351 (2005).

[18] C.-H. Lee, H. Guo, S. Radhakrishnam, A. Lal, C. Szekely, T. Mcclelland, and A. P. Pisano, "A Batch Fabricated Rubidium-Vapor Resonance Cell for Chip-Scale Atomic Clocks," Technical Digest of Solid-State Sensor, Actuator and Microsystems Workshop, Hilton Head Island, SC, June 6-10 (2004).

[19] S. W. Du, M. B. Squires, Y. Imai, L. Czaia, R. A. Saravanan, V. Bright, J. Reichel, T. W. Hansch, and D. Z. Anderson, "Atom-Chip Bose-Einstein Condensation in a Portable Vacuum Cell", Physical Review A, 70, 053606 (2004).

[20] L.-A. Liew, J. Moreland, V. Gerginov, and J. Kitching, To be published. 
[21] Y.-Y. Jau, A. Post, E. Miron, N. Kuzma, and W. Happer, "Physics and Cell Manufacturing of Miniature Atomic Clocks," Workshop on Chip Scale Atomic Clock:

Status and Potentiality, Koganei, Tokyo, Japan, March 9-10 (2006).

[22]N. Cyr, M. Tetu, and M. Breton, "All-Optical Microwave Frequency Standard - a Proposal", IEEE Transactions on Instrumentation and Measurement, 42, 640 (1993).

[23] W. E. Bell and A. L. Bloom, "Optically Driven Spin Precession", Physical Review Letters, 6, 280 (1961).

[24]A. Brannon, J. Breitbarth, and Z. Popovic, "A LowPower, Low Phase Noise Local Oscillator for Chip-Scale Atomic Clocks", IEEE Microwave Theory and Techniques Symposium, in press (2005).

[25] I. Bargatin and M. L. Roukes, "Nanomechanical Analog of a Laser: Amplification of Mechanical

Oscillations by Stimulated Zeeman Transitions", Physical Review Letters, 91, 138302 (2003).

[26] D. Rugar, R. Budakian, H. J. Mamin, and B. W. Chui, "Single Spin Detection by Magnetic Resonance Force Microscopy", Nature, 430, 329 (2004). 